\title{
Artículo
}

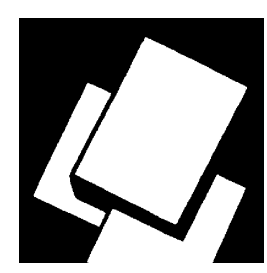

\section{Prepararse para la jubilación: el papel del apoyo social en la gestión de la ansiedad}

\section{Getting ready for retirement: the role of social support in anxiety management}

RITA CHIESA ${ }^{1}$ GUIDO SARCHIELLI ${ }^{1}$

\section{RESUMEN}

La jubilación puede ser definida como una transición que implica el abandono de un rol laboral y, por consiguiente, esto requiere la capacidad de reestructurar el sistema de funciones y actividades personales. Este estudio incluyó 250 empleados de dos administraciones públicas italianas de más de 48 años de edad, con el objetivo de entender cómo durante el tiempo previo a la jubilación el apoyo social recibido por fuentes laborales y extra-laborales puede influir en la ansiedad hacia su jubilación y cómo la última modula la edad a la que se desea jubilarse. Los resultados obtenidos mediante análisis de modelos de ecuaciones estructurales LISREL muestran cómo el apoyo social del superior y la implicación con el trabajo aumentarán la ansiedad debida a la pérdida de la identidad social, mientras que el apoyo de los miembros de la familia tiende a reducir el tamaño de la ansiedad anterior al retiro de la vida laboral. El apoyo de los amigos reduce la ansiedad ante la anticipación de exclusión social. Por lo último, la ansiedad parece influir la edad en la que se desea jubilarse.

\section{ABSTRACT}

Retirement can be considered a transition that implies losing one's work role and therefore there is a need to be capable of restructuring one's systems of personal roles and activities. This study used a sample of 250 employees of public administration aged over 48 to

\footnotetext{
${ }^{1}$ Facoltà di Psicologia, Università di Bologna, Italia.
} 
Prepararse para la jubilación: el papel del apoyo social en la gestión de la ansiedad

find the extent to which work and non-work sources of social support can affect anxiety during the period of preparation for retirement. In addition, the study explored the relation between social support and intended retirement age. LISREL structural equation model analysis shows that supervisor's social support and job involvement increase the anxiety resulting from identity loss, whereas family social support decreases this anxiety. Moreover, friends' social support reduces the anxiety owing to anticipated social exclusion. Finally, retirement related anxiety predicts intended retirement age.

\section{PALABRAS CLAVE}

La jubilación, El apoyo social, La ansiedad.

\section{KEY WORDS}

Retirement; Social support; Anxiety. 


\section{INTRODUCCIÓN}

La necesidad de respuestas políticas y sociales en contra de lo que muchos llaman el "reto demográfico", debido al envejecimiento de la población en los países industrializados, ha llevado a una mayor atención hacia los estudios sobre la jubilación. En particular, la perspectiva psico-social ha supuesto, especialmente durante los últimos veinte años, una importante contribución para la comprensión de las formas en las que las personas se enfrentan a la etapa final de su carrera y su jubilación.

Esta perspectiva se basa en la definición de la jubilación como transición laboral en relación a la elaboración de una carrera de prolongada, con la intención de reducir la intervención psicológica en el trabajo (Feldman, 1994).

Esta definición subraya la importancia implícita de por lo menos dos aspectos en el estudio de la jubilación: el primero es que, siendo una transición que implica la salida de un puesto de trabajo, "La jubilación puede ser vista como una transición que implica la ampliación, la redefinición y el cambio de papeles "(Carter y Cook, 1995, p. 67), el segundo aspecto se refiere a la importancia de considerar la extensión del tiempo de la jubilación, ya que este evento permitirá "un proceso que ocurre durante un período temporal y que requiere tomar decisiones, implementar y llevar las consecuencias" (Beehr, 1986, p.50).

En cuanto al primer punto, la jubilación es diferente de otras transiciones que impliquen el abandono laboral, como también la salida de una carrera o de un trabajo (Adam, Prescher, Beehr y Lepisto, 2002). La jubilación se considera a menu- do un evento caracterizado por la pérdida: la pérdida de trabajo, remuneración e identidad (Bossé, Aldwin, Levenson, Workman-Daniels, y Ekerdt, 1990). Por este motivo, la jubilación supone una importante reestructuración de las funciones que, según Super (1990), definen el espacio de la vida de una persona y, por consiguiente, requiere flexibilidad y capacidad de adaptación a las nuevas condiciones.

Cabe señalar que el impacto la salida del trabajo quedará modulado por diversos factores, tanto psicológicos como contextuales (Van Solinge y Henkens, 2008).

Desde un punto de vista psicológico, el efecto de la pérdida de la función laboral parece vinculada a dos factores: la importancia de la actividad laboral en la definición de la identidad personal y la presencia de otras importantes funciones sociales en la definición de sí mismo, que puede sustituir satisfactoriamente la función laboral (Carter y Cook, 1995).

Según la Work-Role Attachment Theory (Carter y Cook, 1995), el apego a la función laboral puede dificultar la adaptación a la jubilación. Por otra parte, la adaptación a la nueva situación puede ser especialmente difícil para aquellos que muestran una escasa inversión en los papeles extra-laborales en lo que respecta a las relaciones familiares, a la amistad, a la participación en alguna organización y a la dedicación a actividades de esparcimiento, ya que esto limita la presencia de importantes papel extra-laborales para la definición de identidad que puedan sustituir el papel del trabajo abandonado (Atchley, 1971).

La centralidad del trabajo en relación a otros elementos, como la familia, en la 
Prepararse para la jubilación: el papel del apoyo social en la gestión de la ansiedad

definición de la conciencia de sí mismo es muy diferente de una persona a otra, lo que implica que la transición a la jubilación puede requerir diferentes niveles de la reestructuración de sí mismo (Shanahan y Porfelli, 2002).

En lo que respecta a la extensión temporal de la jubilación, se pueden identificar tres etapas principales de desarrollo de este proceso: la de preparación, la de decisiones y la de adaptación (Atchley, 1976).

La etapa de preparación previa a la jubilación coincide con una progresiva clarificación de las actitudes sobre la jubilación y una planificación del tiempo y de la forma de su retiro de la vida laboral. Ekerdt (1998) define este periodo como "la anticipación remota", que es la construcción gradual por parte del individuo del tema de las expectativas que conducen a la salida del trabajo. Según Atchley (1976) durante este período la persona inicialmente muestra una vaga, pero en general positiva actitud hacia la jubilación. En el momento de la jubilación, los planes son más detallados, pero la actitud tiende a degenerar en ansiedad y desesperación por la pérdida de su actividad laboral.

Por supuesto, la fase de preparación para la jubilación tiene un significado personal diferente en función de la historia individual y de la centralidad de la actividad laboral desempeñada.

En general, sin embargo, las expectativas que se forman durante esta fase de previsión son cruciales para determinar las intenciones y la planificación de la retirada y el momento de la verdadera retirada de la vida laboral (Prothero y Beach, 1984); y la planificación aparece estrechamente vinculada a la adaptación de los resultados de la transición (Taylor y Doverspike, 2003): la planificación aumenta la probabilidad de crear expectativas realistas que pueden facilitar la adaptación a la condición de jubilado (Kim y Moen, 2001, Taylor-Carter, Cook, y Weinberg, 1997).

Sin embargo, mientras que la primera socialización, o proceso de entrada, ha sido muy estudiada en el proceso de adquisición de la función laboral en los jóvenes, desde esta perspectiva no se estudiado mucho el proceso de salida, o fase de preparación para la jubilación (Reitzes y Mutran, 2006). La mayor atención se ha centrado en la etapa que coincide con la toma de una decisión de retirarse de la vida laboral. En particular, se han estudiado principalmente tres aspectos de la decisión de jubilarse (Beehr, 1986, Kiefer y Briner, 1998): la relación entre la jubilación y el trabajo, la percepción de la voluntariedad de la elección y la relación entre la jubilación y la edad.

El análisis del primer aspecto se refiere al estudio de las formas de trabajo que persisten después de la jubilación (Weckerle y Shultz, 1999).

El estudio de la percepción de la voluntariedad de la elección se refiere al análisis del efecto de varios factores en tal percepción. Kiefer y Briener (1998) sugieren considerar la voluntariedad como una consecuencia de la presencia de factores de push (de empuje) y pull (de arrastre o atracción): el primero se refiere a los factores negativos laborales y extra-laborales que fomenten el abandono de trabajo, mientras que el segundo se refiere a los factores atractivos de la condición de titular de una pensión. La mayor incidencia de los factores pull en la elección de jubi- 
larse aumenta la percepción de la voluntariedad de la decisión, el que parece un preludio a una mejor adaptación a la condición de jubilado (Reitzes, Mutran, y Fernández, 1996).

El tercer aspecto de la decisión de jubilarse se refiere a cuándo se hace esta elección. La atención a este aspecto ha hecho posible identificar algunas diferencias entre la jubilación anticipada y la jubilación a tiempo (Beehr, 1986).

Por último, la tercera fase de los estudios considerados en el proceso de transición a la jubilación se refiere a la adaptación a la nueva condición social. La fase de adaptación presenta un carácter procesal (Atchley, 1976), y a menudo su estudio se integra con el estudio de la adaptación más generalizada a los cambios debido a su edad avanzada.

Este estudio pretende centrarse en la preparación para la jubilación y, en particular, trata de explorar un aspecto aún no investigado, que es la relación entre el apoyo social, la ansiedad de jubilación y de la edad en la que se desea jubilarse.

\section{EL APOYO SOCIAL Y EL PROCESO DE JUBILACIÓN}

En general, las teorías sobre el desarrollo de las carreras y los estudios sobre el enfrentamiento de la transición de trabajo, consideran el apoyo social un recurso importante para el individuo.

En concreto, el Modelo de Transiciones de Schlossberg (1981) incluye el apoyo social entre las variables que afectan al modo de hacer frente a la transición, definidas por el autor como un "sistema de
4S", a saber: 1) la Situación (situation), que afecta a todos los factores contextuales que caracterizan el momento de la transición (por ejemplo, en el caso de una transición a la jubilación, la legislación en materia de seguridad en vigor); 2) el Sí Mismo (self), que se refiere a todos los recursos personales que faciliten el enfrentamiento (por ejemplo, la auto-eficacia y el optimismo); 3) el Apoyo (support), que describe la presencia de formas de apoyo social, tanto de información como emocional, en el momento de la transición; y 4) las Estrategias (strategies) que se refieren a las estrategias de coping adoptadas (distintas por las estrategias centradas en el problema y las centradas en la resolución de problemas emocionales).

Con respecto a la jubilación, Bossé et al. (1990) han llamado la atención sobre la frecuencia con que se da por sentado que la jubilación supone la pérdida de contactos sociales, pero todavía no está claro el grado de pérdida, tanto en términos de extensión de la red social, como de calidad, de apoyo recibido. Taylor y Doverspike (2003) sugirieron investigar el apoyo social para distinguir las diferentes fuentes (familia, amigos, etc.) y los diferentes tipos (emocional, instrumental, etc.) de apoyo social.

En general, la literatura ha dedicado poco tiempo a la reflexión sobre el efecto de apoyo social en el proceso de jubilación, especialmente en lo que respecta a la fase preparatoria de retirada, porque los estudios que incluyen el apoyo social entre las variables estudiadas se refieren en gran parte a la fase de adaptación a la jubilación. El análisis de apoyo social está, por tanto, con frecuencia presente en el debate sobre la necesidad de reestructurar sus redes sociales que se debe al aban- 
dono de la actividad empresarial y, más en general, está integrado con el estudio de mantenimiento de las redes sociales en la vejez.

Está reconocido que con el adelanto de la edad a que se produce la salida laboral, el sistema de redes sociales en el que la persona se encuentra tiende a cambiar. Según la teoría del apoyo social de Kahn y Antonucci (1980), durante el ciclo vital el individuo debe abandonar diferentes actividades, que implican diferentes relaciones sociales. Desde un punto de vista subjetivo, según los autores, las redes sociales puede ser divididas en tres círculos concéntricos que se caracterizan por la proximidad y el grado de confianza con un individuo: en el círculo interior están las personas más cercanas a la persona (por ejemplo, los padres, el cónyuge, los hijos, etc.), mientras que en el círculo intermedio se encuentran las personas con las que el sujeto muestra una relación bastante profunda, que sean ya amigos, parientes o compañeros de trabajo; finalmente, en el círculo exterior se encuentran los conocidos, con los que la persona se relaciona en forma superficial en el trabajo o el ocio. La transición a la jubilación implica el ajuste de la red social al segundo y al tercer círculo, y las relaciones que caracterizan el círculo próximo de la persona siguen siendo generalmente más estable (Atchley, 1999; Carstensen, 1992, Kahn y Antonucci, 1980). Estos cambios no tienen necesariamente efectos negativos sobre el bienestar; autores como Kahn y Antonucci (1980) consideran que este último está garantizado por la calidad del apoyo recibido y no por su cantidad.

Además, algunos autores creen que la reducción de las redes sociales tiene una función adaptativa de selección (Antonuc- ci y Akiyama, 1991; Carstensen, 1993; Field y Minkler, 1988, O'Connor, 1995). De hecho, según la teoría de la selectividad socio-emotiva (Carstensen, 1993 Carstensen, Isaacowitz, y Charles, 1999) la maduración requiere una selección de objetivos y, por tanto, la adaptación de los contactos sociales al fin de lograr sus objetivos. Según esta teoría, la selección de los objetivos que se tiene que perseguir ocurre en relación con su futura perspectiva temporal: los que interpretan su propio futuro en sentido amplio prefieren objetivos proyectados hacia una satisfacción de largo plazo, para la que pueden actuar unas conductas como, por ejemplo, la inversión en el conocimiento, y el mantenimiento de los contactos sociales fundamentales para el logro de sus objetivos. Al contrario, las personas que evalúan su futuro como emocionalmente limitado prefieren objetivos sociales importantes, tales como la autorregulación emocional y la generativity, concebida como un deseo de dejar una importante contribución a las generaciones futuras y obtener mayor satisfacción de las relaciones que implican una participación emocional (Ryff y Heincke, 1983). Cabe señalar que no hay resultados concluyentes de las investigaciones relacionadas con la reducción de las relaciones sociales debida a la edad (Bossé, et al., 1990; Greller y Richtermeyer, 2006).

Es cierto también que el número de interacciones, que impiden el aislamiento social, y la calidad de esos recursos, que proporcionan el apoyo social a la persona, están asociados con el mantenimiento de un alto nivel de bienestar emocional y con el grado de satisfacción de su vida durante la jubilación (Hong y Duff, 1997, Levitt, Antonucci, Clark, Totton, y Finley, 1985; Warr, Butcher, Callinan y Robertson, 
2004), mientras que la percepción de la vejez como de un período de aislamiento social resulta perjudicial para la satisfacción con su propia vida y para el bienestar emocional (Steverink, Westerhof, Bode, y Dittmann-Kohli, 2001).

Taylor y Doverspike (2003) advierten sobre la necesidad no sólo de investigar el alcance de las redes sociales, sino también de destacar la importancia de profundizar en la comprensión de los efectos positivos y negativos que pueden ejercer. De hecho, al estar asociadas con un buen nivel de confort y satisfacción para sus vidas, las redes sociales existen para servir como canales a través de los cuales la persona puede obtener ayuda en forma instrumental o de apoyo emocional. Un ejemplo de la negatividad que las relaciones sociales pueden suponer en relación a la adaptación es que las investigaciones han demostrado como, durante la vejez, el apoyo social ofrecido por los amigos tiene un efecto más importante sobre el bienestar que el del apoyo familiar (Larson, Mannelli, y Zuzanek , 1986, Lee y Shehan, 1989, O'Connor, 1995), porque la percepción de un alto nivel de apoyo de los miembros de la familia ocurre a menudo en las situaciones en que el individuo se ve obligado a pedir ayuda por causa de unos déficit funcionales causado por el envejecimiento. En tales casos, el apoyo recibido de la familia deteriora el sentido de la responsabilidad y la autonomía del individuo. Por el contrario, el apoyo de amigos a menudo se correlaciona con una vida activa, por lo que la comparación con sus compañeros alienta sentimientos positivos sobre sí mismo (Siebert, Mutran y Reitzes, 1999). Como las relaciones con los colegas puede ser una fuente importante de apoyo social (Howard, Marshall, Rechnitzer, Cunningham, y Donner, 1982;
Mutran y Reitzes, 1981), en algunos casos la pérdida de este contacto se puede vivir como un problema (Moen et al 2000), sobre todo porque esta pérdida no suele ser sustituida por nuevos amigos: los lazos de amistad, en realidad, con el acercamiento de la edad se centran en el conocimiento consolidado en el tiempo (Field y Minkler, 1988).

El efecto positivo del apoyo social sobre el nivel de ajuste a la jubilación, sugiere la hipótesis de que el apoyo social pueda también influir en la fase de preparación para la jubilación, sobre todo porque, como un recurso psico-social, puede facilitar el afrontamiento de la transición y alimentar la prefiguración de expectativas positivas para el retiro (Greller y Richtermeyer, 2006); en realidad, sin embargo, como ya se ha previsto, son escasas las investigaciones que han estudiado este aspecto.

Desde la perspectiva de la planificación de la jubilación, es útil distinguir las fuentes de apoyo social en términos de relaciones en el entorno del trabajo y relaciones en el contexto exterior.

Henkens y Tazelaar (1997), examinando la decisión de jubilarse, se dieron cuenta de que desarrollar importantes relaciones en el lugar de trabajo contribuye a evaluar positivamente la oportunidad de seguir trabajando en lugar de jubilarse.

El efecto de las relaciones sociales construidas en el contexto laboral sobre la modalidad y el calendario de preparación y adopción de decisiones relacionadas con la jubilación han sido más a menudo estudiadas en términos negativos. En otras palabras, la relación negativa con los colegas y los superiores se han mencionado 
como posibles factores o condiciones que aceleren la salida del mercado laboral (Beehr, 1986; Beehr, Glazer, Nielson, y Farmer, 2000, Taylor y Shore, 1995). Otra confirmación indirecta de los efectos perjudiciales de la falta de apoyo social en el desarrollo de la carrera se refiere a estudios sobre los estereotipos y la discriminación que sufren los "viejos trabajadores", que pueden conducir a la retirada del trabajo (Chiu, Chan, Snape, y Redman, 2001; Finkelstein y Burke, 1998, Finkelstein, Burke, y Raju, 1995; Henkens, 2000, Lyon y Pollard, 1997; Maurer, 2001, Maurer y Rafuse, 2001; Schellenberg y Silver, 2004; Szinovacz, 2003; Taylor y Walker, 1994, 1998, Wrenn y Mauer, 2004).

En cuanto al efecto del apoyo social a partir de fuentes extra-laborales en la planificación de la jubilación, las principales indicaciones proceden de los estudios que han introducido el estado civil como una variable que afecta al la decisión personal de la retirada del trabajo, y en particular de las investigaciones que han profundizado el proceso de decisión de retirarse como una elección de pareja (Henkens, 1999; Talaga y Beehr, 1995; Smith y Moen, 2004; Kim y Moen , 2002; VanSolinge y Henkens, 2005; Pienta y Hayward, 2002). Esta interdependencia de las decisiones de la pareja se debe no sólo a la evaluación financiera ?por ejemplo, de acuerdo con Clark y McDermed (1986), si tienen un cónyuge que proporciona buenos ingresos aumenta la probabilidad de retirada, especialmente para las mujeres?, sino también a decisiones que afectan al equilibrio entre los roles de la pareja y al intercambio de tiempo entre ellos.

En cuanto a la división de funciones dentro de la pareja, a pesar del aumento gradual de la participación femenina en el mercado de trabajo, el hombre aceptará más raramente las situaciones en las que su esposa sigue trabajando aún después de su propia retirada de la vida laboral (Szinovacz y DeViney, 2000). Si esto sucede, los conflictos familiares aumentarán drásticamente (Hofmeister y Moen, 1998, Myers y Booth, 1996; Szinovacz, 1996; Szinovacz y Schaffer, 2000).

Las parejas donde ambos cónyuges son jubilados se caracterizan por un mayor bienestar psicológico y una mayor satisfacción de la vida conyugal en la jubilación, sobre todo cuando los dos muestran una fuerte inversión en su vida social, incluso en el período previo a la jubilación

(Atchley, 1992; Kelly y Westcott, 1991; Reitzes, Mutran y Fernández, 1996). De hecho, en las situaciones en las que la relación matrimonial es positiva, la jubilación puede ser vista como una manera de recuperar el tiempo para compartir con la pareja las actividades de ocio; en este sentido, la pareja puede ser alentada a planear su jubilación de manera simultánea (Honig 1996; Honig y Hanuch 1985; Pienta 1999; Pienta y Hayward, 2002; Ruhm, 1996; Van-Solinge y Henkens, 2005).

En cualquier caso, la literatura carece de ejemplos de evaluación directa del efecto del apoyo social extra-laboral, familiar y de los amigos en los planes de jubilación: de aquí se desprende la necesidad de realizar más estudios.

\section{OBJETIVOS E HIPÓTESIS DEL ESTUDIO}

Fletcher y Hansson (1991), en la definición de ansiedad por la jubilación como 
"un sentimiento generalizado de aprensión o preocupación por las consecuencias inciertas, imprevistas y potencialmente destructivas de la inminente jubilación" (p.77), asocian la ansiedad con el temor de fracaso en comparación con las dos principales tareas de desarrollo resultantes de la transición a la jubilación: el enfrentamiento de la pérdida de relaciones interpersonales y actividades sociales relacionadas con el contexto laboral; y el desarrollo y la inversión en nuevas relaciones interpersonales y actividades sociales extra-laborales.

Este estudio quiere verificar cómo el apoyo social recibido durante la etapa previa a esta transición por diversas fuentes, dos en el contexto laboral (superiores y compañeros) y dos fuera de este contexto (familia y amigos), respectivamente, afectan al componente social de la ansiedad hacia la jubilación, y cómo la última influye en la definición de la edad ideal para jubilarse.

Por lo que se refiere al apoyo recibido en el contexto laboral, se considera que éste puede ayudar al individuo a alimentar una visión positiva de su trabajo (Beehr, 1986; Beehr et al., 2000, Taylor y Shore, 1995) y que, en consecuencia, puede aumentar la ansiedad hacia la jubilación como un acontecimiento que genera la salida de un contexto gratificante. En concreto, se supone que:

Hipótesis 1: El efecto del apoyo recibido por su superior en relación a la ansiedad de jubilarse sea mediado por la implicación con el trabajo: se supone que un superior que tiene mucho cuidado para motivar a sus empleados al job involvement y que la implicación con el trabajo, como índice de identificación personal con su propio trabajo, tengan un efecto positivo para la ansiedad hacia la jubilación, debida a la percepción individual de pérdida de su propia identidad social (Fletcher y Hansson, 1991).

Hipótesis 2: Teniendo en cuenta la frecuencia con la que las relaciones con colegas generan relaciones de amistad (Howard Marshall, Rechnitzer, Cunningham, y Donner, 1982; Mutran y Reitzes, 1981), se supone que el apoyo recibido por los colegas pueda aumentar la ansiedad debida a la anticipación de la exclusión social, ligada a su condición de titular de una pensión.

En cuanto a la ayuda social por parte de las fuentes extra-laborales, la literatura ya ha demostrado en otros contextos su papel como recurso de coping (Thoits, 1986), capaz de reducir el estado de ansiedad (Zimet, Dahlem, Zimet, y Farley, 1988 ) y los estudios sobre la jubilación han destacado la importancia de la familia como una fuente de identidad alternativa al trabajo (Carter y Cook, 1995; Reitzes, Mutran, y Fernández, 1996) y la importancia de las redes de amistad como fuente de integración social (Siebert, Mutran, y Reitzes, 1999). Por ello se parte del supuesto de que:

Hipótesis 3: El apoyo de los miembros de la familia reduce la ansiedad hacia la jubilación y, en particular, la ansiedad relacionada con la pérdida de la identidad social (h3a), porque se supone que la percepción de apoyo de la familia se unirá a la percepción de desempeñar un papel importante para la propia identidad dentro de ésta (Carter y Cook, 1995; Reitzes, Mutran, y Fernández, 1996) y se supone también que el apoyo de los miembros de la familia aumente la adaptación social a la condición de pensionista, porque que 
tranquilizaría al individuo sobre la probabilidad de tener cerca de sí personas que comparten su tiempo libre (h3b).

Hipótesis 4: De acuerdo a las investigaciones que muestran el efecto del apoyo de amigos para el bienestar en la vejez (Larson, Mannelli, y Zuzanek, 1986, Lee y Shehan, 1989, O'Connor, 1995), se supone que el apoyo recibido reduzca la ansiedad ligada a la anticipación de la exclusión social (h4a) y aumente la prefiguración de una buena adaptación a la condición de jubilado (h4b). la pérdida de identidad social (h5b) (Fletcher y Hansson, 1991), aumenta la edad a la que el individuo quiere jubilarse, mientras que la expectativa de la adaptación social reduce la edad a la que el individuo desea jubilarse (h5c).

El modelo en su totalidad se resume en la Figura 1. Además de la hipótesis de la relación causal, la figura muestra también la correlación aparente entre las dos fuentes de apoyo social en el contexto laboral (superiores y colegas), y entre las dos fuentes de apoyo extra-laborales (la familia y los amigos).

Figura 1. Relaciones supuestas entre el apoyo social, la ansiedad de la edad efectiva de jubilación y la elegida por el individuo para retirarse de trabajo

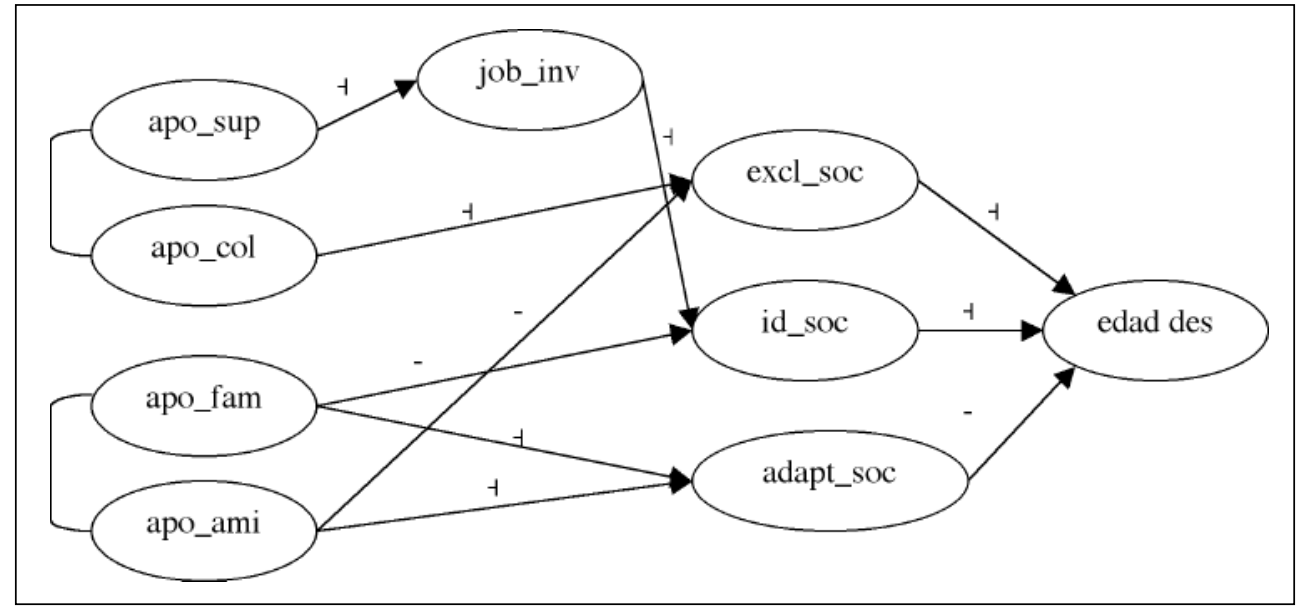

Leyenda: apo_sup=apoyo social del superior; apo_col= apoyo social de los colegas; apo_fam=apoyo social de la familia; apo_ami=apoyo social de los amigos; job_inv=job involvement; excl_soc=previsión de la exclusión social; id_soc=amenaza a la identidad social; adapt_soc=adaptación social.

Por último, se estima que durante el período de preparación para la jubilación la ansiedad puede afectar a la planificación de esta transición (Prothero y Beach, 1984). En concreto, se supone que: Hipótesis 5: La ansiedad social hacia la jubilación debido a la anticipación de exclusión social (h5a) y la percepción de

\section{MÉTODO}

\section{Procedimiento y características de los participantes}

La investigación ha sido realizada en dos administraciones públicas, una administración provincial y una municipal, del 
centro-norte de Italia, la dirección de las cuales se ha puesto a disposición para promover la encuesta y distribuir un cuestionario a sus empleados. Dada la gran similitud de las dos administraciones en términos de estructura orgánica, funciones y competencias que participan en ellas, durante la verificación de hipótesis se ha considerado que los participantes conforman una muestra única. Se pidió a las organizaciones que proporcionaran la lista de empleados que habían completado por lo menos 48 años de edad, lo que supuso 273 empleados de la administración provincial y 294 empleados comunales. Aunque no fue posible obtener de las organizaciones datos completos del resto de los empleados, la Dirección de ambas organizaciones ha estimado que la población que tiene más de 48 años representa aproximadamente la mitad de los empleados.

Debido a necesidades específicas de organización no ha sido posible estandarizar los métodos de distribución en las dos organizaciones: la provincia ha permitido ponerse directamente en contacto con los involucrados en la investigación y estar presente durante la recogida de datos para proporcionar cualquier aclaración; mientras que el municipio ha considerado más adecuado entregar el cuestionario a través del sistema de correo interno.

El cuestionario fue acompañado de una carta de presentación de la finalidad de investigación y información sobre la manera de devolverlo. Con el fin de garantizar el anonimato necesario, para la recogida de cuestionarios cumplimentados se preparó también una urna colocada en un punto de cruce para los empleados. Los distintos modos de administración del cuestionario son probablemente la principal razón de la diferente tasa de respuesta registrada en las dos organizaciones: mientras que el número de cuestionarios devueltos de la provincia ascendió a 142 (que ha alcanzado alrededor de un 52\% la tasa de respuesta) la tasa de respuesta comunal se situó en el $37 \%$, con un total de 108 cuestionarios cumplimentados. El total de la muestra es, por consiguiente, de 250 personas.

Los entrevistados son en su mayoría mujeres $(58 \%)$, y tienen edades comprendidas entre 48 y 67 años $(M=54,09, d t=$ $3,71)$. Sólo el $16 \%$ dijo que su estado civil era soltero, mientras que el $84 \%$ eran personas casadas/convivientes. La forma más extendida de contrato es un contrato de duración indeterminada $(90 \%)$, aunque parece que el $6 \%$ de los encuestados tiene un contrato temporal, mientras que el $4 \%$ es empleado a tempo parcial.

La dedicación laboral semanal es de tiempo completo para el $87 \%$ mientras que el $13 \%$ está empleado a tiempo parcial. En comparación con la categoría de contrato del actual gobierno italiano, los encuestados son en su mayoría clasificados en un nivel alto: sólo el 0,5\% pertenece al Grado A (que es la categoría más baja), el $17 \%$ al B, el $53 \%$ al Grado $\mathrm{C}$ y el $19 \%$ a la categoría $\mathrm{D}$, mientras que el restante $10,5 \%$ está en la posición de gerente.

En cuanto a la antigüedad en su trabajo los entrevistados se diferencian muchísimo unos de otros: la persona con menos años de carrera dijo llevar 7 años trabajando, en comparación a los 47 años reportados por la persona con mayor antigüedad laboral $(M=30,91 ; d t=5.75)$, pero parece aun mayor la diferencia interindividual en relación a los años que la persona pasó trabajando en la organización de la que ahora forma parte. La relación de trabajo 
con su organización va de un mínimo de un año a un máximo de 44 años $(M=$ $20,21 ; d t=10,73)$.

\section{Instrumentos}

El cuestionario contaba con instrucciones para ser auto-cumplimentado. Contiene dos partes: la primera dirigida a la investigación de las construcciones psicológicas, la segunda consiste en un registro. En la primera parte, para simplificar la recopilación y la forma de respuesta, las distintas escalas han sido estandarizadas a una escala de Likert de cinco puntos, siendo el extremo inferior 1 (totalmente en desacuerdo) y el superior 5 (completamente de acuerdo).

Apoyo social percibido. Se ha investigado la percepción de apoyo social recibido de diferentes fuentes, dos en relación con las relaciones existentes en lugar de trabajo con los colegas y con el supervisor, y dos sobre la dimensión extra-laboral, la familia y los amigos. La percepción del apoyo recibido en el trabajo de la parte del superior y de los colegas ha sido investigada a través de dos dimensiones (item 9) extractas del Job Content Questionnaire de Karasek (1985), en la versión italiana de Cenni y Barbieri (1997). El cuestionario completo investiga el entorno psico-social del mundo laboral. El análisis factorial exploratorio ha confirmado la presencia de dos factores (varianza explicada $=65,49 \%$ ). Los factores sociales que pertenecen la ayuda recibida del superior (item 4; ejemplo de item "Mi jefe inmediato está interesado en el bienestar de sus empleados") y el apoyo social recibido de colegas (5 item; ejemplo de item "La gente con la que trabajo ha establecido conmigo una relación personal también"). La percepción de apoyo de la familia y de los amigos se ha investigado a través de una adaptación de la Multidimensional Scale of Perceived Social Support. Esta escala fue validada en una muestra de adolescentes (Zimet et al., 1988). Se encontró después una amplia aplicación en los estudios de coping. La escala fue traducida al italiano con el método de traducción de back translation. La redacción original de la escala consta de 12 elementos que identifican tres fuentes de apoyo social: familia, amigos, una persona importante. En este estudio, hemos optado por no incluir la tercera dimensión, vinculada sobre todo a los tipos de relaciones de adolescentes, y que ya ha demostrado durante la validación de Zimet et al. (1988) una correlación bastante alta con el apoyo social de amigos. En cambio, la independencia entre la percepción del apoyo recibido de los miembros de la familia y el de los amigos se ve confirmada por los estudios que han utilizado otras escalas (Ir y Heller, 1983). Los items se han modificado a fin de medir el apoyo de la familia y lo de los amigos hacia el mundo laboral. El análisis factorial exploratorio realizado sobre los 8 item seleccionados ha confirmado la presencia de dos factores (varianza explicada $=74,37 \%$ ): el apoyo recibido de los miembros de la familia (item 4, por ejemplo el item: "Mi familia me ayuda a tomar decisiones que afectan a mi carrera ") y el apoyo recibido de amigos (4 item, ejemplo del item: "Yo tengo amigos con quien compartir alegrías y penas").

La consistencia interna parece buena para todas las dimensiones: el apoyo del superior $(\alpha=, 88)$, lo de los colegas $(\alpha=83)$, el apoyo familiar $(\alpha=91)$ y el apoyo de los amigos $(\alpha=, 84)$.

Job Involvement. La implicación con el trabajo, definida como una identificación 
psicológica con un contexto laboral específico, fue investigada a través de la reducción a 6 items del Job Involvement Questionnaire (Kanungo, 1982). La escala fue traducida al italiano con el método de back translation. El análisis factorial exploratorio ha confirmado la graduación mono-factorial (varianza explicada = $56,84 \%$ ) (Ejemplo de item: "El enlace con mi trabajo es tan fuerte que sería muy difícil para mí romperlo”). El índice de consistencia interna parece satisfactorio $(\alpha=.84)$.

Ansiedad ante la jubilación. La ansiedad hacia la jubilación ha sido investigada mediante los componentes sociales de la Social Components of Retiremen Anxiety Scale (Fletcher y Hansson, 1991) de 23 items, que explora la ansiedad social hacia la jubilación, que es el conjunto de expectativas y sentimientos negativos en comparación con las consecuencias interpersonales de la inminente jubilación. La escala fue traducida al italiano con el método de back translation.

El análisis factorial exploratorio ha encontrado una estructura con tres factores (varianza explicada $=50,73 \%)$, en parte coincidentes con el estudio sobre la escala original, que ha determinado la presencia de cuatro factores que se pueden definir como: Social Integration and Identity, Social Adjustment/Hardliness, Anticipated Social Exclusion, Lost Friendship. El análisis factorial sugiere la supresión de dos item, con baja saturación $(<.40)$ en el factor.

En este estudio, los factores que han sido utilizados son: Anticipación de la exclusión social (11 item, por ejemplo el tema: "Cuando me jubile voy a estar solo”), Pérdida social (5 ítem, por ejemplo el item: "No me puedo imaginar sin trabajo"); y Adaptación Social (5 item, por ejemplo el item: "Jubilarme me permitirá hacer las cosas con los amigos que yo no puedo hacer durante el trabajo"). El análisis de consistencia interna muestra índices satisfactorios para todas las tres dimensiones: la ansiedad en anticipación de la exclusión $(\alpha=, 89)$; ansiedad debida a la amenaza para la identidad social $(\alpha=.75)$; y la adaptación social $(\alpha=, 71)$.

Edad ideal para la jubilación. Un solo item se ha empleado para investigar la edad ideal para la jubilación. La redacción del item es: “¿A qué edad querría Usted (o hubiera querido) jubilarse?" Aunque la literatura no recomienda el uso de un solo item en la construcción de modelos estructurales (Bollen, 1989), en este caso, el único item respeta la representación semántica entre el indicador y la construcción del contenido

Datos de Registro. En la segunda parte del cuestionario, se pedía que fueran indicados: género, edad, estado civil, experiencia en el empleo, organización del retiro, el tipo de contrato (fijo, de colaboración) y la dedicación semanal (tiempo completo o parcial); y categoría contractual (A, B, C, D, Director).

\section{Análisis de Datos}

Después del análisis factorial exploratorio (Análisis de Componentes Principales con rotación Varimax) y el análisis de consistencia interna (alpha de Cronbach) para comprobar la estructura de la escala, se ha continuado con la verificación de hipótesis mediante análisis multivariante de datos realizado por el paquete Lisrel 8.7 . 
Prepararse para la jubilación: el papel del apoyo social en la gestión de la ansiedad

La elección de utilizar el análisis de modelos de ecuaciones estructurales (SEM) se debe a dos razones: en primer lugar para encontrar la confirmación de la estructura factorial de las escalas utilizadas, que sólo en algunos casos se han traducido directamente del inglés para la presente investigación; el segundo propósito es verificar la influencia mutua de las diversas variables que definen la experiencia de apoyo social y la anticipación de la jubilación durante la etapa avanzada de la carrera.

Dado el tamaño actual de la muestra, es preferible proceder con el método de desglose parcial que permite reducir el número de parámetros estimados (Bagozzi y Edwards, 1998) agregando al azar los temas relativos a la construcción de la misma (Perugini y Conner, 2000). El ajuste global de los modelos se evaluó por medio de cuatro índices: el test de Chi cuadrado $\left(\chi^{2}\right)$; el índice de aproximación cuadrática media (RMSEA, Steiger, 1989); el índice de ajuste comparativo (CFI, Bentler, 1989, 1990); y el índice de ajuste no-normalizado (NNFI, Tucker y Lewis, 1973; Bentler y Bonnet, 1980).

A diferencia de $\chi^{2}$, los otros índices se muestran menos sensibles al tamaño de la muestra y al número de variables observadas y por este motivo, en caso de pequeño tamaño de la muestra y de presencia de muchas variables observadas, estos reducen el riesgo de subestimar el ajuste del modelo y aceptar una hipótesis nula (Bentler, 1990). El índice RMSEA se considera aceptable cuando toma valores inferiores a .08. El CFI y el NNFI son valores que están entre 0 y 1 : la proximidad a 1 indica una buena adaptación del modelo (Bentler, 1990; Bentler y Bonnet, 1980). Convencionalmente se considera un buen modelo con CFI y NNFI superior o igual a .95 (Hu y Bentler, 1999), aunque algunos autores consideran aceptables valores superiores a .90 (Hair, Anderson, Tatham, e Black, 1992).

Para probar la hipótesis de la mediación (h1) se comparó el modelo con path de mediación con los modelos adicionales que incluyen el path directo (Perugini y Conner, 2000): como la inclusión del path directo ha mejorado el ajuste del modelo mismo, es decir la diferencia entre ${ }^{-}$Ç relativos a los dos modelos fue importante, la hipótesis de mediación ha sido descartado y no hay verificación de cada una de las condiciones descritas por Baron y Kenny (1986) para definir la mediación.

\section{RESULTADOS}

La tabla 1 muestra los resultados descriptivos y de correlación entre las variables tomadas en consideración. En relación con el contexto laboral, la principal fuente de apoyo social es proporcionada por colegas $(\mathrm{t}(234)=5,84, \mathrm{p}<.001)$, mientras que en el contexto extra-laboral es la familia la que puede asegurar el mayor apoyo $(\mathrm{t}(236)=6,60, \mathrm{p}<.001)$, aunque la desviación típica relativa al apoyo de los miembros de la familia indica una mayor diferenciación intersubjetiva de la muestra en conexión con en esta variable.

La ansiedad hacia la jubilación es reducida: la prefiguración de la adaptación social es en realidad mayor que la ansiedad relativa a la pérdida de identidad social $(\mathrm{t}(241)=6,50, \mathrm{p}<.001) \mathrm{y}$, sobre todo, a la anticipación de la exclusión social $(\mathrm{t}(236)=16,25, \mathrm{p}<.001)$. Esta última también es más baja que la por la pér- 
dida de identidad social $(\mathrm{t}(234)=-18.90$, $\mathrm{p}<.001)$. La media de edad identificada como ideal para jubilarse es de alrededor de 57 años. variable asumida como mediadora no ha sido posible verificar la importancia del path directo del apoyo del superior hacia la ansiedad debida a la pérdida de la iden-

Tabla 1. Medias, desviaciones típicas y correlaciones de Pearson

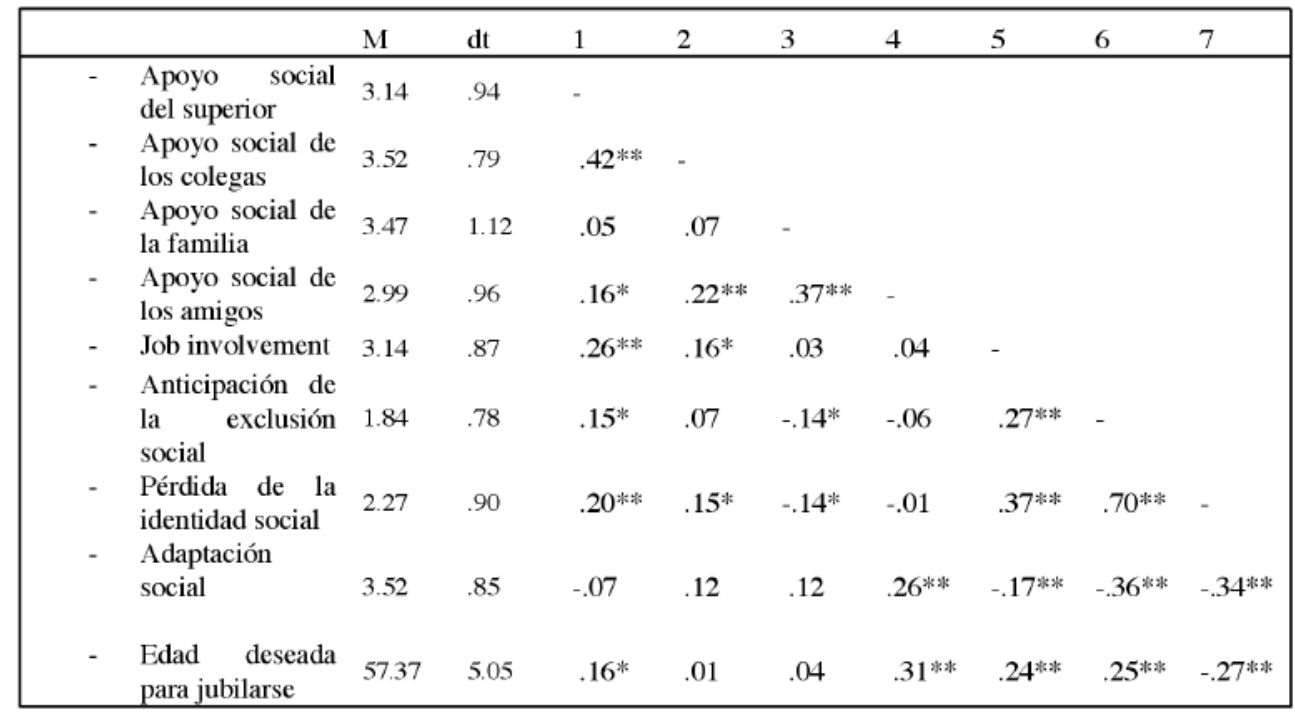

Nota: $\mathrm{N}=250 ; * \mathrm{p}<.05 ; * * \mathrm{p}<.01$

Como para el modelo general, los índices de ajuste parecen aceptables $\left(\chi^{2}=\right.$ 622,49, df $=264, \mathrm{p}<.001 ;$ RMSEA $=$. 074, $\mathrm{CFI}=.92$; NNFI =. 91). El modelo de mediación confirma la estructura identificada por el análisis factorial exploratorio tanto para variables exógenas, como para las variables endógenas; todas cargas factoriales son significativas y los errores resultan aceptables.

Continuando con la verificación de hipótesis, con respecto a la hipótesis 1 , la implicación con el trabajo debe mediar la participación de la relación entre el apoyo recibido de su superior y la ansiedad por la pérdida de la identidad social debida a la jubilación; sin incluir en el modelo la tidad $\operatorname{social}\left(\gamma_{\text {std }}=.19, \mathrm{p}<.01\right)$. Por lo tanto, se ha procedido en la validación de la hipótesis de mediación, comparando el modelo mediado con el modelo en el que se ha colocado el path directo: la diferencia entre $\chi^{2}$ aparece significativa $\left(\Delta \chi^{2}=7.28, \mathrm{df}=1 ; \mathrm{p}<.01\right), \mathrm{y}$ entonces, como el path directo muestra que se puede mejorar el modelo, se rechaza la idea de la mediación.

Contrariamente a la hipótesis, el apoyo social del superior tiene un efecto directo tanto en la implicación con el trabajo $\left(\gamma_{\text {std }}=.27, \mathrm{p}<.01\right)$ como en la ansiedad debida la pérdida de la identidad sociales ligada a la jubilación $\left(\beta_{\text {std }}=.42\right.$, $\mathrm{p}<.01)$. 
Prepararse para la jubilación: el papel del apoyo social en la gestión de la ansiedad

El último modelo presentado en la Figura 2 también incluye la ruta directa entre el superior y el apoyo de ansiedad por la pérdida de identidad social $\left(\chi^{2}=\right.$ 615,21 , df $=263, \mathrm{p}<.01$, RMSEA $=.074$, $\mathrm{CFI}=.92$; NNFI $=.91$ ).

En cuanto a la hipótesis 2, la influencia del apoyo recibido de colegas en la anticipación de la exclusión social debido a la jubilación no es significativa. Como consecuencia, la hipótesis 2 no ha sido confirmada.

En cuanto a las fuentes extra-laborales de apoyo social, así como exige hipótesis 3 , el apoyo de los miembros de la familia muestra un efecto negativo en la ansiedad debida a la pérdida de la identidad social $\left(\gamma_{\text {std }}=-.19, \mathrm{p}<.01\right)$ (h3a confirmada $)$ mientras que ningún efecto significativo de la ayuda de miembros de la familia sobre la dimensión social del ajuste (h3b no confirmada).
En cuanto al apoyo recibido de los amigos, éste no parece influir en la anticipación de la exclusión social (h4a no confirmada), pero muestra un efecto positivo importante sobre la adaptación social y sustancial de la jubilación $\left(\gamma_{\text {std }}=.38, \mathrm{p}\right.$ $<.01$ ) (h4b confirmada).

En cuanto a la ansiedad social hacia la jubilación, el efecto de anticipación de la exclusión social no es significativo (h5a no confirmada), y la ansiedad por la pérdida de identidad social y de adaptación social muestran, respectivamente, un efecto positivo $\left(\beta_{\text {std }}=.28, \mathrm{p}<.01\right)(\mathrm{h} 5 \mathrm{~b}$ no confirmada) y un efecto negativo $\left(\beta_{\text {std }}=\right.$ $.24, \mathrm{p}<.01)$ (h5c confirmada) en relación a la edad en la que se desea jubilarse (h5c).

\section{DISCUSIÓN}

En relación con la etapa de jubilación objeto de este trabajo, es decir, la fase de

Figura 2. Modelo estructural testado

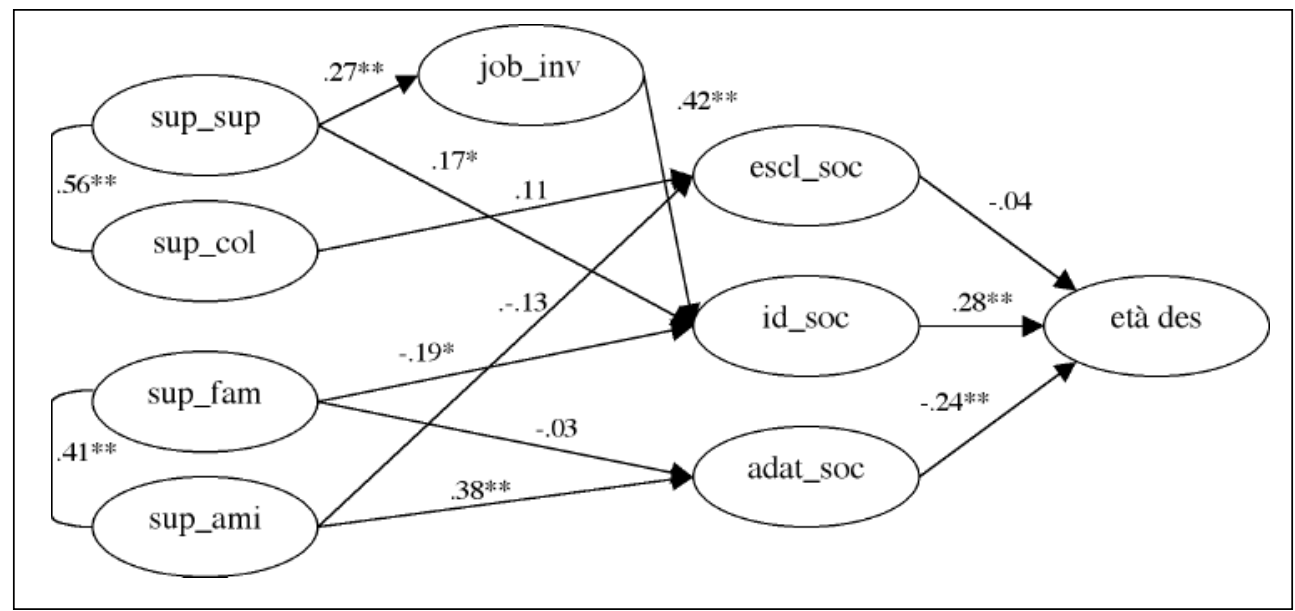

Leyenda: apo_sup=apoyo social del superior; apo_col= apoyo social de los colegas; apo_fam=apoyo social de la familia; apo_ami=apoyo social de los amigos; job_inv=job involvement; excl_soc=previsión de la exclusión social; id_soc=amenaza a la identidad social; adapt_soc=adaptación social. 
preparación para la jubilación, este estudio ha producido algunos resultados interesantes.

En primer lugar, los resultados confirman que, si el retiro tiene las características de transición voluntaria y anticipada, que complementa el desarrollo natural de su carrera de aprendizaje a lo largo de la vida, la fase de preparación para la transición no es especialmente problemática: los resultados muestran un nivel de ansiedad reducido y un relativamente alto nivel de expectativas positivas con respecto a la posibilidad de adaptarse a la condición de persona retirada.

En otras palabras, en la anticipación que precede a una jubilación voluntaria, el abandono del trabajo es visto como una oportunidad para recuperar el tiempo para cultivar los intereses y las relaciones sociales sacrificadas por los compromisos que figuran en este trabajo.

Este estudio también muestra el efecto de la ansiedad por la pérdida de la identidad social debida al abandono de la función social de las empresas y la previsión de los efectos de ajuste a la edad de jubilación prefiguración en la que se desea jubilarse, lo que confirma el efecto de las expectativas antes de la retirada de las decisiones relacionadas con la jubilación (Flecther y Hansson, 1991; Henkens, 1999).

Además, como demuestran las investigaciones recientes sobre la relación positiva entre la ansiedad antes de la retirada, en particular relacionados con la pérdida de contactos sociales, y la dificultad de adaptación después de la retirada (Van Solinge y Henkens, 2008), estos resultados permiten suponer para el futuro un buen nivel de adaptación de los encuestados a la condición de titular de una pensión.

Este estudio también ayuda a especificar el efecto de un recurso importante como es el apoyo social, relativo al establecimiento de expectativas para la jubilación y, en particular, a la regulación de la ansiedad antes de la retirada. En cuanto a la ayuda social recibida de fuentes en el lugar de trabajo, puede ser incluida entre las variables relativas al mundo laboral la planificación de la jubilación (Beehr, 1986; Beehr, et al, 2000, Taylor y Shore, 1995). Los resultados de este estudio destacan el importante papel desempeñado por el apoyo prestado del superior: de hecho, éste es crucial para motivar a la participación en su trabajo, pero, a diferencia de lo que se supone, que también muestra un efecto directo sobre el tamaño de ansiedad acerca de la pérdida de la identidad social debida a la jubilación. Esto sugiere un papel importante de reconocimiento social a los supervisores, lo que estimula aún más el estudio, destinado principalmente al desarrollo de las políticas de gestión de los recursos humanos, con especial atención a las políticas encaminadas a la gestión de los trabajadores de más edad.

Por lo que se refiere, sin embargo, a la relación entre el apoyo recibido de colegas y la ansiedad debida a la anticipación de la exclusión social en la jubilación, los resultados no apoyan la hipótesis. Considerando, sin embargo, que todas las suposiciones sobre la exclusión social no han sido confirmadas, el hallazgo sugiere la posibilidad de que la medida pueda no reflejar adecuadamente la construcción de esta dimensión.

Desplazando la atención a las fuentes de apoyo extra-laborales, el estudio cons- 
tata una importante contribución de la familia en la regulación del nivel de ansiedad para la jubilación. En particular, el apoyo de los miembros de la familia parece reducir el temor de la pérdida de identidad social, lo que confirma la idea de la Work-Role Attachment Theory (Carter y Cook, 1995) acerca de los efectos sobre la adaptación a la jubilación como resultado de la inversión en papeles extra-laborales personales, como familia.

En contraste, los datos no confirman la hipótesis sobre el efecto del apoyo de los miembros de la familia en la adaptación a la jubilación: esto podría ser debido a que el papel familiar es una importante fuente de identidad social en la jubilación, pero a veces, entraña obligaciones y compromisos que no permiten la planificación de muchas actividades de ocio en la jubilación; por ejemplo, el cuidado de padres ancianos que ya no son autosuficientes y que en muchos casos se les llama a las personas adultas.

Respecto a la anticipación de la adaptación social, sin embargo, se muestra el efecto positivo del apoyo que el individuo recibe de los amigos. Este resultado está en consonancia con lo que se ha destacado en la literatura sobre la importancia de las relaciones de amistad en la definición de la adaptación a la condición de jubilado (Larson, Mannell, y Zuzanek, 1986, Lee y Shehan, 1989, O'Connor, 1995).

El apoyo de amigos determina los prolegómenos de la reestructuración de las actividades de ocio y las relaciones sociales, y, a diferencia de lo que supone, no parece afectar significativamente a la ansiedad de la anticipación de la exclusión social. Como ya se ha mencionado, el resultado podría estar afectado por la medida utilizada para esta dimensión de la ansiedad.

\section{CONCLUSIONES Y LIMITACIONES}

En general, se puede concluir diciendo que este estudio ayudará a definir las características que tiene la fase de preparación de la transición a la jubilación.

En primer lugar, este estudio pone de manifiesto el importante papel del apoyo social como un recurso para la planificación de la jubilación. Este resultado, si bien recogido en un contexto de retiro voluntario, puede proporcionar una importante materia de reflexión para aquellos que, encargándose de la gestión de los recursos humanos, están obligados a acompañar a las personas en procesos complejos y, a veces, problemáticos como los provocados por el abandono de su rol laboral por una jubilación anticipada. En estas circunstancias, es importante ayudar a la persona a ser consciente de sus recursos, y por lo tanto, del apoyo social que recibe, para poder prever una estrategia para superar la transición de trabajo.

Al mismo tiempo, los resultados de la influencia de la ayuda proviene de fuentes del contexto laboral y extra-laboral confirman la necesidad de considerar la jubilación como una transición que involucra no sólo al ámbito de la vida profesional, pero que implica también la necesidad de modificar el equilibrio entre las esferas de la vida. En otras palabras, existe la necesidad de incluir estudios sobre la jubilación en los estudios sobre el desarrollo de la carrera con el fin de comprender en mayor profundidad la forma en la que la persona gestiona el proceso de reestructuración de 
sí misma y de su sistema de papeles activado por la jubilación. Este estudio es fundamental para determinar cuales necesidades de apoyo al proceso de career selfmanagement pudieran surgir durante la transición a la jubilación.

En este sentido, una limitación de la investigación que puede ser identificada es el disponer sólo de un índice (apoyo social) para determinar la influencia de las redes y de los roles sociales de la persona en el proceso de anticipación de la jubilación; y de manera similar, para investigar la planificación sólo se dispone del índice relativo a la edad considerada conveniente para la jubilación.

La investigación futura, por lo tanto, podrá ampliar el debate introducido por estos resultados iniciales mediante la inclusión, con un diseño de investigación longitudinal, de la dimensión temporal relativa a la manera de desarrollar y promover la planificación en el tiempo previo a la jubilación.

Por otra parte, la inversión futura en la investigación podría ayudar a superar otra limitación de este estudio: la generalización de los resultados. Aunque en este estudio la referencia al contexto de trabajo sólo esté relacionada con el apoyo recibido, las normas de desarrollo de la carrera profesional en el contexto de la administración pública a la que pertenecen los encuestados hacen muy previsible la programación de la carrera profesional y la de jubilación. La baja incertidumbre podría explicar la formación de expectativas positivas que se verifican hacia la jubilación. Por esta razón, sería conveniente buscar la confirmación de los resultados obtenidos replicando el estudio en otros contextos laborales.

\section{REFERENCIAS}

Adam, G.A., Prescher, J., Beehr, T.A. \& Lepisto, L. (2002). Applying WorkRole Attachment Theory to retirement decision-making. International Journal of Aging and Human Development, 54(2), 125-137.

Antonucci, T.C. \& Akiyama, H. (1991). Convoys of social support: Generational issues. En S.K. Pfeifer y M.B Sussman (Eds.), Marriage and Family Review, 16 (1,2), 103-124.

Atchley, R.C. (1971). Retirement and leisure participation: Continuity or crisis? The Gerontologist 11(1), 13-17.

Atchley, R.C. (1976). The sociology of retirement. New York: John Wiley.

Atchley, R. C. (1992). Retirement and marital satisfaction. En E.M. Szinovacz \& E.D. J. Ekerdt (Eds.), Families and retirement (145-158). Newbury Park, CA: Sage.

Atchley, R.C. (1999). Continuity and adaptation in aging. London: The John Hopskins University Press.

Bagozzi, R.P. \& Edwards, J.R. (1998). A General Approach for Representing Constructs in Organizational Research. Organizational Research Methods, 1 (1), 45-87.

Baron, R.M. \& Kenny, D.A. (1986). The moderator-mediator variable distinction in social psychological research: Conceptual, strategic, and statistical considerations. Journal of Personality and Social Psychology, 51, 1173-1182.

Beehr, T.A. (1986). The process of 
Prepararse para la jubilación: el papel del apoyo social en la gestión de la ansiedad

retirement: A review and recommendation for future investigation. Personnel Psychology, 39 (1), 31-55.

Beehr, T.A., Glazer, S., Nielson, N.L. \& Farmer, S.J. (2000). Work and nonwork predictors of employees' retirement ages. Journal of Vocational Behavior, 57(2), 206-225.

Bentler P.M. (1989). EQS structural equations program manual. Los Angeles: BMDP Statistical Software.

Bentler P.M. (1990). Comparative fit index in structural models. Psychological Bullettin, 107 (2), 238-246.

Bentler, P.M. \& Bonnet, D.G. (1980). Significance tests and goodness of fit in the analysis of covariance structures. Psychological Bulletin, 88 (3), 588-606.

Bollen, K.A. (1989). Structural Equations with Latent Variables. New York, NY: Wiley.

Bossé, R., Aldwin, C., Levenson, M.R., Workman-Daniels, K. \& Ekerdt, D. (1990). Differences in social support among workers and retirees: Findings from the Normative Aging Study. Psychology and Aging, 5 (1), 41-47.

Carstensen, L.L. (1992). Social and emotional patterns in adulthood: Support for socioemotional selectivity theory. Psychology and Aging, 7 (3), 331-338.

Carstensen, L.L. (1993). Motivation for social contact across the life span: A theory of socioemotional selectivity. In J.E. Jacobs (Ed.) Nebraska symposium on motivation: 1992, Developmental Perspectives on Motivation, (Vol. 40, 209 -
254). Lincoln: University of Nebraska Press.

Carstensen, L.L., Isaacowitz, D. \& Charles, S.T. ( 1999). Taking time seriously: A theory of socioemotional selectivity. American Psychologist, 54 (3), 165181

Carter, M. \& Cook, K. (1995). Adaptation to retirement: Role changes and psychological resources. The Career Development Quarterly, 44 (1), 67-82.

Cenni, P. \& Barbieri, F. (1997). Karasek's Job Content Questionnaire: Una versione italiana tradotta e adattata. Report di ricerca non pubblicato

Chiu, W., Chan, A., Snape, E. \& Redman, T. (2001). Age Stereotypes and Discriminatory Attitudes towards Older Workers: An East-West Comparison'. Human Relations, 54(5), 101-133.

Clark, R.L. \& McDermed, A.A. (1996). Earning and pension compensation: The effect of eligibility. Quarterly Journal of Economics, 101, 341-361

Ekerdt, D.J. (1998). Workplace norms for the timing of retirement. En K.W. Schaie y C. Schooler (Eds.), Impact of work on older adults (101-121). New York: Springer Publishing Company.

Feldman, D.C. (1994). The decision to retire early: a review and conceptualization. Academy of Management Review, 19(2), 285-311.

Field, D. \& Minkler, M. (1988). Continuity and change in social support between young-old, old-old, and very-old 
adults. Journal of Gerontology, 43 (4), 100-106.

Finkelstein, L. M. \& Burke, M. J. (1998). Age stereotyping at work: The role of rater and contextual factors on evaluations of job applicants. The Journal of General Psychology,125 (4), 317-345.

Finkelstein, L.M., Burke, M.J. \& Raju, N.S. (1995). Age discrimination in simulated employment contexts: An integrative analysis. Journal of Applied Psychology, 80 (6), 652-663.

Fletcher, W. L., e Hansson, R.O. (1991). Assessing the social components of retirement anxiety. Psychology and Aging, 6 (1), 76-85.

Greller, M.M. \& Richtermeyer, S.B. (2006). Changes in social support for professional development and retirement preparation as a function of age. Human Relations, 59(9), 1213-1234.

Karasek, R. (1985). Job Content Questionnaire. Los Angeles: Department of Industrial and Systems Engineering, University of Southern California.

Hair, J.F. Jr, Anderson, R.E., Tatham, R.L. \& Black, W.C. (1998). Multivariate Data Analysis (5th ed.). Upper Saddle River, NJ: Prentice-Hall.

Henkens, K. (1999). Retirement intentions and spousal support: A multi-actor approach. Journal of Gerontology: Social Sciences, 54B (2), 63-73.

Henkens, K. (2000). Supervisors' attitudes about early retirement of subordinates. Journal of Applied Social Psychology, 30 (4), 833-852.
Henkens, K. \& Tazelaar, F. (1997). Explaining Retirement. Decisions of Civil Servants in the Netherlands. Research. on Aging, 19(2), 13-173.

Hofmeister, H. \& Moen, P. (1998). Long Term Employment and the Marital Quality of Retirees: Links Through Gender Roles. BLCC Working Paper (98108), Cornell, NY: Cornell Employment and Family Careers Institute.

Hong, L.K. \& Duff, R.W. (1997). Relative importance of spouses, children and friends in the life satisfaction of retirement community residents. Journal of Clinical Geropsychology, 3 (4), 275-282.

Honig, M. (1996). Retirement expectations: Differences by race, ethnicity, and gender. The Gerontologist, 36, 373-382.

Honig, M. \& Hanuch, G. (1985). Partial Retirement as a Separate Mode of Retirement Behavior. Journal of Human Resources, 20, 21-46.

Howard, J. H., Marshall, J., Rechnitzer, P. A., Cunningham, P. A. \& Donner, A. (1982). Adapting to retirement. Journal of the American Geriatrics Society, 30, 488500 .

Hu, L., e Bentler, P.M. (1999). Cutoff criteria for fit indexes in covariance structure analysis: Conventional criteria versus new alternatives. Structural Equation Modeling, 6(1), 1-55.

Kahn, R.L. \& Antonucci, T.C. (1980). Convoy over the life course: Attachment, roles and social support. En P.B. Baltes y O. Brim (Eds.), Life-span development and behavior (Vol. 3, 254-283). New York: Academic Press. 
Prepararse para la jubilación: el papel del apoyo social en la gestión de la ansiedad

Kanungo, R.N. (1982). Measurement of job and work involvement. Journal of Applied Psychology, 67 (3), 341-349.

Kelly, J. R. \& Westcott, G. (1991). Ordinary retirement: Commonalities and continuity. International Journal of Aging and Human Development, 32, 81-89

Kiefer, T. \& Briner, R.B. (1998). Managing Retirement Rethinking links between Individual and Organization. European Journal of Work and Organizational Psychology, 7(3), 373-390.

Kim, J.E. \& Moen, P. (2001). Moving into retirement: Preparation and transitions in late midlife. En M.E. Lachman, (Ed). Handbook of midlife development (487-527). Hoboken, NJ: John Wiley \& Sons, Inc.

Kim, J. E., \& Moen. P. (2002). Retirement transitions, gender, and psychological well-being: A life-course, ecological model. Journal of Gerontology: Psychological Sciences, 57B (3), P212-P222.

Larson, R., Mannell, R. \& Zuzanek, J. (1986). Daily well-being of older adults with friends and family. Psychology and Aging, 1 (2), 117-126.

Lee, G. \& Shehan, C. (1989). Retirement and marital satisfaction. Journal of Gerontology, 44(6), 226- 230

Levitte, M.J., Antonucci, T.C., Clark, M.C., Totton, J. \& Finley, G.E. (1985). Social support and well-being: preliminary indicators based on two samples of elderly. International Journal of Aging Human Development, 21, 61-77.

Lyon, P. \& Pollard, D. (1997). Percep- tions of the older employee: is anything really changing? Personnel Review, 26 (4), 245-257.

Maurer,T.J. (2001).Career-relevant learning and development, worker age, and beliefs about self-efficacy for development. Journal of Management, 27(2), 123-140.

Maurer,T.J. \& Rafuse, N. E. (2001). Learning, not litigating: managing employee development and avoiding claims of age discrimination. Academy of Management Executive, 15, 110-121.

Moen, P. \& Smith, D. (2004). Retirement satisfaction for retirees and their spouses: Do gender and the retirement decision-making process matter? Journal of Family Issues, 25(2), 262-285.

Moen, P., Erickson, M.A. \& DempsterMcClain, D. (2000). Social Role Identities among Older Adults in a Continuing Care Retirement Community. Research on Aging, 22, 559-579.

Mutran, E. \& Reitzes, D.C. (1981). Retirement, identity and wellbeing: realignment of role relationship. Journal of Gerontology, 36 (6), 733-740.

Myers, S. M. \& Booth, A. (1996). Men's Retirement and Marital Quality. Journal of Family Issues, 17, 336-358.

O'Connor, B.P. (1995). Family and friend relationships among older and younger adults: Interaction motivation, mood, and quality. International Journal of Aging and Human Development, 40 (1), 9-29.

Perugini, M. \& Conner, M. (2000). Pre- 
dicting and understanding behavioural volitions: The interplay between goals and behaviours. European Journal of Social Psychology, 30, 705 - 731.

Pienta, A. M. (1999). Early Childbearing Patterns and Women's Labor Force Behavior in Later Life. Journal of Women \& Aging, 11 (1), 69-83.

Pienta, A.M. \& Hayward, M.D. (2002).Who expects to continue working after age 62 ? The retirement plans of couples. Journal of Gerontology, 57B (4), 199-208.

Prothero, J. \& Beach, L.R. (1984). Retirement decision: Expectation, intention, action. Journal of Applied Social Psychology, 14, 162-174.

Reitzes, D.C. \& Mutran, E.J. (2006). Lingering identities in retirement. Sociological Quarterly, 47(2), 333-359.

Reitzes, D.C., Mutran, E.J. \& Fernandez, M.E. (1996). Does retirement hurt wellbeing? Factors influencing selfesteem and depression among retirees and workers. Gerontologist, 36 (5), 649-656.

Ryff, C.D. \& Heincke, S.G. (1983). Subjective organization of personality in adulthood and aging. Journal of Personality and Social Psychology, 44 (4), 807-816.

Ruhm, C. J. (1996). Gender differences in employment behavior during late middle life. The Journal of Gerontology, 51B (1), 11-17

Schellenberg, G. \& Silver, C. (2004). You can't always get what you want: retirement preferences and experiences.
Canadian Social Trends. Ottawa: Statistics Canada.

Schlossberg, N.K. (1981). A model for analysing human adaptation to transitions. The Counseling Psychologist, 9 (2), 2-18.

Shanahan, M.J. \& Porfelli, E. (2002) Integrating the life course and life-span: Formulating research questions with dual points of entry. Journal of Vocational Behavior, 61(3), 398-406.

Siebert, D.C., Mutran, E.J. \& Reitzes, D.C. (1999). Friendship and social support: The importance of role identity to aging adults. Social Work, 44(6), 522-533.

Steiger, J.H. (1989). EzPATH: A supplementary module for SYSTAT and SYGRAPH. Evanston: SYSTAT.

Steverink, N., Westerhof, G.J., Bode, C. e Dittmann-Kohli, F. (2001). The personal experience of aging, individual resources and subjective well being. Journal of Gerontology, 56B(6), 364 -373.

Super, D.E. (1990). A life span, lifespace approach to career development. En D. Brown, L. Brooks \& Associates (Eds), Career choice and development (2nd ed.) (197-261). San Francisco: Jossey-Bass.

Szinovacz, M. E. (1996). Couple's employment/retirement patterns and marital quality. Research on Aging, 18 (2), 243-268.

Szinovacz, M. (2003). Contexts and Pathways: Retirement as Institution, Process, and Experience. En G. A. Adams y T.A. Beehr (Eds.), Retirement: reasons, processes and results (6-52). New York: Springer Publishing. 
Prepararse para la jubilación: el papel del apoyo social en la gestión de la ansiedad

Szinovacz, M. E. \& De Viney, S. (2000). Marital characteristics and retirement decisions. Research on Aging, 22, 470-489.

Szinovacz, M. \& Schaffer, A. M. (2000). Effects of retirement on marital conflict management. Journal of Family Issues, 21 (3), 367-389

Talaga, J.A. \& Beehr, T.A. (1995). Are there gender differences in predicting retirement decisions? Journal of Applied Psychology, 80(1), 16-28.

Taylor-Carter, M. A., Cook, K.\& Weinberg, C. (1997). Planning and expectations of the retirement experience. $E d u$ cational Gerontology, 23 (3), 273-288.

Taylor, M. A. \& Doverspike, D. (2003). Retirement planning and preparation. In A. Adams \& T. A. Beehr (Eds.), Retirement: Reasons, processes, and results (53-82). New York: Spring Publishing.

Taylor, M.A. \& Shore, L.M. (1995). Predictors of planned retirement age: An application of Beehr's model. Psychology and Aging, 10 (1), 76-83.

Taylor, P. \& Walker, A. (1994). The ageing workforce: Employers' attitude towards older people. Work, Employment and Society, 8 (4), 569-591.

Taylor, P. \& Walker, A. (1998). Employers and older workers: Attitudes and employment practices. Ageing and Society, 18 (6), 641-658.

Thoits P.A. (1986). Social support as coping assistance. Journal of Consulting and Clinical Psychology, 54 (4), 416-423.

Tucker, L.R. \& Lewis, C. (1973). A reliability coefficient for maximum likelihood factor analysis. Psychometrika, 38, 1-10.

VanSolinge, H. \& Henkens, K. (2005). Couples' adjustment to retirement: a multi-actor panel study. Journal of Gerontology: Social Sciences, 60B (1), 11-20

Van Solinge, H. \& Henkens, K. (2008). Adjustment to and satisfaction with retirement: two of a kind? Psychology and aging, 23(2), 422-434.

Warr, P., Butcher, V., Robertson, I. \& Callinan, M. (2004). Older people's wellbeing as a function of employment, retirement, environmental characteristics and role preference. British Journal of Psychology, 95 (3), 297-324.

Weckerle, J.R. \& Shultz, K.S. (1999). Influences on the bridge employment decision among older USA workers. Journal of Occupational and Organizational Psychology, 72(3), 317-329.

Wrenn, K.A. \& Maurer, T.J. (2004). Beliefs about older workers learning and development behavior in relation to beliefs about malleability of skills, agerelated decline, and control. Journal of Applied Social Psychology, 34 (2), 223242.

Zimet, G.D., Dahlem, N.W., Zimet, S.G. \& Farley, G.K. (1988). The Multidimensional Scale of Perceived Social Support. Journal of Personality Assessment, 52 (1), 30-41. 